\title{
EL PLANTEO Y RESOLUCIÓN DE PROBLEMAS DESDE DOMINIOS DISCRETOS Y PROFESORES DE MATEMÁTICAS EN FORMACIÓN
}

\author{
THE POSING AND SOLVING OF PROBLEMS \\ FROM DISCRETE DOMAINS AND \\ MATHEMATICS TEACHERS IN TRAINING
}

\author{
Luis Fernando Mariño \\ Rosa Virginia Hernández² \\ Víctor Julio Useche Arciniegas ${ }^{3}$ \\ Universidad Fancisco de Paula Santander
}

\section{RESUMEN}

Este escrito reporta los hallazgos de una investigación con un enfoque cualitativo y un diseño desde la teoría fundamentada, centrado en responder la pregunta, ¿Cómo son las estrategias manifestadas por un grupo

$\overline{1}$ Doctor en Educación Matemática (Universidad Antonio Nariño, Colombia). Magister en Educación Matemática. Licenciado en Matemáticas y Computación. Profesor Departamento de Matemáticas y Estadística de la Universidad Francisco de Paula Santander (Cúcuta). Correo. fernandoml@ufps.edu.co. Orcid: https://orcid. org/0000-0002-3438-6963

2 Magister en Educación Matemática. Licenciada en Matemáticas y Computación. Profesora adscrita al Departamento de Matemáticas y Estadística de la Universidad Francisco de Paula Santander (Cúcuta). Correo. rosavirginia@ufps.edu. Orcid: /orcid.org/0000-0002-2638-671X

3 Maestría en Física. Ingeniero Civil. Profesor adscrito al Departamento de Física de la Universidad Francisco de Paula Santander (Cúcuta). Correo. victorjulioua@ufps. edu.co. Orcid: /orcid.org/0000-0003-0635-6676 de profesores de matemáticas en formación cuando plantean y resuelven problemas desde situaciones en dominios discretos? Como fuentes de datos se diseñaron e implementaron una serie de secuencias de aprendizaje y una entrevista semiestructurada. Los hallazgos muestran como las tareas propuestas en las secuencias de aprendizaje, posibilitaron que cada participante construyera relaciones, hiciera afirmaciones, a partir de esas afirmaciones, generalizarayutilizara ejemplos y contraejemplos como forma de prueba. Se destaca también, la manera como los estudiantes solucionaron los problemas cuando se imponían nuevas exigencias a las soluciones de la ecuación lineal diofántica de la forma $a x+b y=c$ y la forma como las utilizaron posteriormente para plantear 
nuevos problemas y proponer situaciones de la vida real como contextos para formular nuevos problemas. Como resultado se construyó el núcleo de la teoría caracterizado como un flujo de acciones e interacciones entre las estrategias de conjeturar, generalizar, probar y plantear problemas. Finalmente, esto condujo a que el planteo de problemas está inmerso en la resolución de problemas desde el contexto propuesto y que los estudiantes son una buena fuente para plantear problemas.

\section{PALABRAS CLAVE}

Planteo de Problemas, Resolución de problemas, Teoría Fundamentada, Dominios Discretos.

\section{ABSTRACT}

This paper reports the findings of a research with a qualitative approach and a grounded theory design, focused on answering the question, how are the strategies manifested by a group of $\begin{array}{lllll}5 & 3 & 5 & \text { mathematics teachers in training when they pose }\end{array}$ and solve problems from situations in discrete domains? A series of learning sequences and a semi-structured interview were designed and implemented as data sources. The findings show how the tasks proposed in the learning sequences made it possible for each participant to build relationships, make assertions, generalize from these assertions, and use examples and counterexamples as a form of proof. The way in which the students solved the problems when new demands were imposed on the solutions of the linear diophantine equation of the form

$a x+b y=c$ and the way in which they subsequently used them to pose new problems and propose real life situations as contexts to formulate new problems is also highlighted. As a result, the core of the theory was constructed, characterized as a flow of actions and interactions between the strategies of conjecturing, generalizing, testing and posing problems. Finally, this led to the conclusion that problem posing is immersed in problem solving from the proposed context and that students are a good source for problem posing.

\section{KEY WORDS}

Problem Posing, Problem Solving, Grounded Theory, Discrete Domains.

\section{INTRODUCCIÓN}

La resolución de problemas ha centrado la atención de investigadores en educación matemática en las últimas décadas, en contraste, el planteo (invención o formulación) de problemas ha sido poco abordado. En el currículo de la matemática escolar hay una larga historia, que muestra aportes significativos en aspectos afectivos, cognitivos y metacognitivos acerca de la resolución de problemas. Desde otra mirada, para algunos investigadores, el planteo de problemas es considerado como un área de investigación relativa y aparentemente nueva. Sin embargo, y como lo afirman Ellerton, Singer y Cai (2015), en 1987, Henry Belfield, en su libro Revised Model Elementary Arithmetic, sugería a los profesores, que orientaran y dejaran que los niños plantearan sus propios problemas. Mientras que Polya (1945), en su libro How to solve, aunque no aparece explicito, afirma que el planteo de problemas ocurre y está inmerso en la resolcución de problemas.

Desde comienzos de este siglo, algunos paises como China y Estados Unidos, lideran y hacen esfuerzos por incorporar el planteo de problemas en el curriculo de matemáticas en diferentes niveles educativos y la formación de profesores de matemáticas en este tema (Cai, Hwang, Jiang, y Silber, 2015; Cai, y otros, 2020). Estos esfuerzos evidencian el interés de muchos investigadores y educadores matemáticos, por llevar y hacer que el planteo de problemas, tenga un papel relevante, como estrategia de aprendizaje, además de orientar e inducir al 
estudiante en el desarrollo de su pensamiento matemático.

Desde otra mirada, en las concepciones tradicionales y contemporáneas, tanto en matemática como en educación matemática, prevalece la idea, de que un buen problema lo plantea un matemático experto, un profesor con experiencia o proviene de un libro de texto y la tarea del estudiante, es resolver el problema (Kilpatrick, 1987; Cai, Hwang, Jiang y Silber, 2015). En contraste, y como lo afirman Felmer, Pehkonen y Kilpatrick (2016), las altas demandas cognitivas que exige el planteo de problemas, puede proporcionar contextos intelectuales que enriquecen el desarrollo del pensamiento matemático del estudiante.

El constructo teórico problema ha sido caracterizado de diversas maneras. Siguiendo la definición clásica de Kupisiewicz (1964), conceptualmente se entiende por problema, una dificultad teórica o práctica que provoca una actitud indagadora de una persona enriqueciendo sus conocimientos. Para Mayer (2010), un problema se produce cuando una situación está en un estado, el resolutor intenta y quiere llevarla a otro estado, pero hay una serie de obstáculos, que no permite transitar con facilidad entre estos estados.

En lo que respecta a la resolución de problemas, para Polya (1981) resolver un problema, es como encontrar una salida a una dificultad, es una forma de sortear un obstáculo. Mientras que para Mayer (2010), la resolución del problema se produce cuando el solucionador del problema se compromete con una actividad cognitiva dirigida a superar el problema. Para este autor, la resolución de problemas es un resumen de los procesos cognitvos centrados en el cambio del estado dado, al estado final donde el procedimiento de solución no es obvio.

Desde lo cognitivo, Polya (1945) sugirió cuatro fases como marco para la resolución de problemas: entender el problema, diseñar un plan, llevar a cabo el plan y mirar hacia atrás en el trabajo. Schoenfeld (2016) por su parte, caracterizó cinco dimensiones que intervienen directa, dinámica e inter-relacionadamente: a) dimensión cognitiva, base de conocimientos, b) heurísticas, estrategias en la resolución de problemas, c) dimensión meta cognitiva, monitoreo y control (auto-regulación), d) dimensión afectiva, creencias y afectos, y e) práctica matemática. Entre tanto, Mason, Burton y Stacey (2010), propusieron tres fases en la resolución de problemas: entrada, ataque y revisión. En este mismo sentido Mayer (2010), caracteriza la resolución de problemas en dos grandes fases, que las denominó representación del problema y solución del problema.

Por otra parte, en los últimos años, el interés por incorporar el planteo de problemas en la enseñanza de las matemáticas en los diferentes niveles educativos ha aumentado considerablemente en investigadores $y$ profesores de matemáticas. Esto se debe, a un creciente reconocimiento de que las actividades inmersas en el planteo de problemas pueden, promover el desarrollo conceptual de los estudiantes, mejorar su capacidad de comprensión, fomentar su capacidad de razonar y de comunicarse matemáticamente (Cai, Jiang, Hwang, Nie y Hu, 2016), además de despertar su interés y curiosidad (Cai, Hwang, Jiang y Silber, 2015).

Para Cai y Hwang (2020), el planteo de problemas "es el proceso de formular $y$ expresar un problema dentro del dominio de las matemáticas" (p. 2). Cai J., Hwang S., Jiang C., Silver S. (2015), afirman que:

$$
\begin{aligned}
& \text { Por planteo de problemas } \\
& \text { en la educación matemática, } \\
& \text { nos referimos a varios tipos } \\
& \text { de actividades relacionadas } \\
& \text { que implican o apoyan a los }
\end{aligned}
$$


profesores y estudiantes que formulan (o reformulan) y expresan un problema o tarea basado en un contexto particular (al que nos referimos como el contexto del problema o situación del problema), (p.2).

Siguiendo a Cai y Hwang (2020), Cai et al. (2015), Xu, Cai, Liu y Hwang (2019), el planteo de problemas se refiere a las tareas, como aquellas que requieren que los profesores o los estudiantes generen nuevos problemas y preguntas basadas en situaciones dadas, ya sea como expresiones o diagramas matemáticos. Estos autores caracterizan el planteo de problemas, como un conjunto de tres actividades intelectuales específicas: a) Los propios profesores plantean problemas matemáticos sobre la base de situaciones determinadas o de expresiones o diagramas matemáticos, b) los profesores predicen los tipos de problemas que pueden plantear los estudiantes sobre la base de situaciones determinadas, expresiones o diagramas matemáticos, y c) los profesores diseñan tareas de planteo de problemas matemáticos para que los estudiantes planteen problemas.

Entre tanto, investigadores como Leung (2016), Kilpatrick (1987), Felmer, Pehkonen y Kilpatrick (2016) afirman que el planteo es inseparable de solución. Mientras que, para Silver (2013), el planteo de problemas implica la generación de nuevos y nuevas preguntas. Brown y Walter (1983) por su parte, proponen como estrategia que a partir de un problema conocido hacer modificaciones acerca de, ¿que pasa sí?, lo que ellos denominaron reformular el problema.

Generalmente, las investigaciones que describen y aportan en estrategias, tanto en el planteo como en la resolución de problemas, han surgido de una diversidad de situaciones en dominios continuos. En contraste y aunque parezca sencillo plantear y resolver problemas que involucran ecuaciones lineales diofánticas de la forma $a x+b y=c$ es una tarea interesante y retadora para el estudiante, que exige intuición y creatividad. Este tipo de ecuaciones se trabajan desde dominios discretos (los números enteros) y las soluciones también ocurren en este mismo dominio.

Este reporte, forma parte de una investigación más amplia, que tiene como propósito aportar avances en la caracterización del planteo y resolución de problemas desde la variación y el cambio. Específicamente, se muestran los hallazgos que responden a la pregunta: ¿Cómo son las estrategias manifestadas por un grupo de profesores de matemáticas en formación cuando plantean y resuelven problemas desde situaciones en dominios discretos?

\section{METODOLOGÍA}

La pregunta de investigación acerca de ¿cómo son las estrategias manifestadas por el grupo de estudiantes?, condujo a la adopción de un enfoque cualitativo. Como lo afirma Ernest (1997), el enfoque de investigación cualitativo en educación matemática se ocupa de la comprensión humana, la interpretación, la intersubjetividad y la verdad vivida y tiene como propósito registrar los fenómenos en términos de comprender las manifestaciones de los participantes. Esto es consecuente con el propósito del estudio centrado en interpretar, dar sentido y caracterizar los datos manifestados por los participantes.

Participantes. Estuvo conformado por un grupo de 15 estudiantes que tomaron un curso de Teoría de Números, durante el segundo semestre del año 2020. El $78 \%$ por ciento de los participantes fueron hombres y el $22 \%$ mujeres, sus edades oscilaban entre los 18 y 23 años de edad. Los participantes fueron elegidos por conveniencia, 
puesto que uno de los investigadores es el profesor que orienta la materia.

\section{LAS FUENTES DE DATOS Y SU DINÁMICA DE TRABAJO.}

Como técnicas para la recolectar los datos se diseñaron y rediseñaron tres secuencias de aprendizaje y una entrevista semiestructurada retrospectiva. Las secuencias de aprendizaje tuvieron como propósito, orientar a los participantes en la búsqueda de relaciones y a construir sus propias estrategias para plantear y resolver problemas, en situaciones que involucraron ecuaciones lineales diofánticas de la forma $a x+b y=c$ desde la variación y el cambio.

La intención siempre fue, orientar al estudiante para que pensara matemáticamente.

Esta dinámica, se mantuvo en el diseño y realización de la entrevista, que se fundamentó

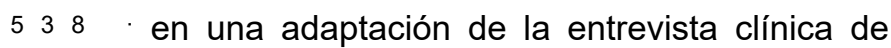
Piaget (Ginsburg, 1981). La intención o propósito estuvo centrada en que fuera el estudiante quien construyera, relaciones y estrategias matemáticas desde su propia perspectiva y no fuera el profesor quien les diera las soluciones, como fórmulas a seguir. Esta estrategia es consecuente con el constructivismo, más específicamente con el constructivismo radical, que se fundamenta en que: la función de la cognición es adaptativa y sirve para organizar el mundo experiencial, no al descubrimiento de una realidad ontológica (Von Glasersfeld, 2013).

\section{ESTRATEGIA DE INVESTIGACIÓN.}

Para poder cumplir el propósito de la investigación, se diseñó y siguió una estrategia desde la teoría fundamentada. Siguiendo a Charmaz (2006, 2014), Corbin y Strauss (2008, 2017), las investigaciones que siguen este enfoque, se caracterizan por: a) un tabajo paralelo entre la recolección y análisis de datos, b) los códigos y categorías se construyen desde los datos, c) el muestreo y saturación teórica $y$, d) el proceso siempre está permeado por el método de comparación constante. Como resultado, la teoría se va construyendo a medida que transcurre la investigación y no al final de la recolección de datos, como ocurre tradicionalmente en otros escenarios.

De allí que, la estrategia estuvo conformada por un proceso ciclico compuesto por tres fases. Fase 1, se implementó la primera secuencia de aprendizaje, se seleccionan los primeros incidentes y se inició el proceso de codificación abierta. Como resultado de esta fase surgen los primeros códigos, que sirvieron como base para la revisión, analisis y rediseño de la segunda secuencia de aprendizaje. Fase 2, segunda intervención, con la segunda secuencia de aprendizaje, continua el proceso de codificación abierta y axial, surgieron de allí las primeras categorías. Fase 3 , se diseña y rediseña la tercera secuencia de aprendizaje y la entrevista semiestructurada, esto permitió recolecar más datos que posibilitaron saturar la teoría; como resultado de esta fase, surgieron las categorías centrales que representaron la teoría formal desde los datos.

\section{LOS PROCESOS SIMULTÁNEOS DE RECOLECCIÓN, CODIFICACIÓN, ANÁLISIS DE DATOS Y LA CONSTRUCCIÓN INDUCTIVA DE LA TEORÍA.}

Siguiendo lo expresado en el párrafo anterior, aunque la estrategia la determinaron tres fases, el proceso simultáneo de recolección y análisis de datos, parece lineal, pero en realidad no lo fue. El trabajo se caracterizó por un proceso cíclico de ida y vuelta permanente a medida que se recolectaban más datos y el análisis inmediato de éstos, para elaboración de códigos y categorías, que posibilitaron elaborar las categorías centrales, cuando los investigadores consideraron que se había saturado la teoría. 
Fase 1. Al implementar la primera secuencia de aprendizaje se propuso a los participantes que plantearan y resolvieran un problema que involucrara la ecuación lineal diofántica $6 x+21 y=102$. La primera acción de los participantes fue inventar problemas en el contexto de los números reales. Curiosamente, ninguno de los participantes pudo realizar esta tarea, posiblemente debido al tema era nuevo para ellos, además de trabajar siempre en el contexto de los números reales.

Ante esta situación, el profesor investigador, sugiere a los estudiantes una serie de cuestiones, para resolver primero la ecuación. Por ejemplo: Cuestión 1: reemplace las variables $x$ e $y$, por números enteros, que hagan verdadera la ecuación, con estos valores construya una tabla con tres columnas, la primera columna para los valores de $x$, la segunda para los valores de $y$, la tercera para verificar la solución. Cuestión 2, revise y analice la columna de los números asignados a la variable, ¿qué relación o patrón encuentra en estos números?, haga lo mismo con la columna de los números de la variable . Cuestión 3, ¿qué relación o qué condiciones considera usted que deben imponerse sobre los números $a, b$ y $c$ para que se cumpla la igualdad?

La Tabla 1, muestra las acciones realizadas por los participantes codificados con SA1 P1 y SA2 $\mathrm{P} 3$, al asignar números a las variables $x$ e $y$ que hicieron verdadera la ecuación $6 x+21 y=102$. $S A 1$, significa secuencia de aprendizaje uno, mientras $\mathrm{P} 1$, hace referencia al participante.
Tabla 1. Respuestas SA1 P1 Y SA1 P3

\begin{tabular}{|c|c|c|c|}
\hline \multicolumn{2}{|c|}{ SA1 P1 } & \multicolumn{2}{c|}{ SA2 P3 } \\
\hline$x$ & $y$ & $x$ & $y$ \\
\hline 10 & 2 & 3 & 4 \\
\hline 24 & -2 & -4 & 6 \\
\hline-4 & 6 & -11 & 8 \\
\hline 38 & -6 & -18 & 10 \\
\hline 52 & 10 & -25 & 12 \\
\hline 66 & -14 & 10 & 2 \\
\hline
\end{tabular}

El trabajo de estos dos participantes, junto a otros cuatro fueron seleccionados como los primeros incidentes. Incidentes en el sentido que realizaron un trabajo mejor elaborado (aunque no definitivo) que sus compañeros. Se analizó cada palabra, frase, signo, símbolo y expresiones matemáticas en la totalidad de la secuencia de cada incidente, con la intención de interpretar y dar sentido a las representaciones escritas, manifestadas por cada participante.

A partir de estos datos, surgieron los primeros códigos. Se muestran algunos ejemplos, acerca de la forma como fueron analizados, junto a los conceptos asignados, sus propiedades y dimensiones. Ante la Cuestión 2, que sugería que el participante analizara la columna de los números dados a la variable $x$, la columna de la variable por separado, luego analizarlas conjuntamente en la búsqueda de relaciones P3, afirma:
$x$ tiene una variación de 7 unidades, con respecto a una encontrada, tiene una variación de 2 unidades, respecto a una encontrada. Si en $x$ se suma en $y$ se resta.

Interpretación y sentido dado a los datos: el participante $\mathrm{P} 3$, reconoce un patrón para los diferentes valores de la variable $x$ (van variando de 7 unidades) y la variable $y$ (una variación de 2 unidades). Al mismo tiempo establece 
una relación, esas variaciones, son respecto a un valor o número conocido. Elabora también, una relación de variación simultanea de cambio entre los números asignados a las dos variables: mientras que una aumenta la otra disminuye.

Ante, estas mismas cuestiones el participante codificado como P1, afirma:

\begin{abstract}
Según la columna " $x$ " el patrón que tiene es que a cualquier valor encontrado de " $x$ " que cumpla la igualdad se le adiciona o se le disminuye "7" y al valor de " $y$ " sí a " $x$ " se le adiciona " $y$ " se le disminuye y si a " $x$ " se le disminuye "7" a " $y$ " se le adiciona
\end{abstract}

Interpretación y sentido dado a los datos: P1, establece con claridad que hay un patrón en la forma en que cambia cada una de las variables. Especifica también otra relación, en cuanto a la forma en que cambia independientemente cada una de las variables y como lo hacen simultáneamente. Aunque no lo hacen explicito, para ambos estudiantes es necesario, la existencia de una solución conocida y a partir de las relaciones encontradas se pueden hallar más números que hacen verdadera la ecuación.

El código combinar. Tanto de las manifestaciones escritas de estos dos participantes y los demás seleccionados como incidentes, surge el primer código que se denominó combinar, sus propiedades, dimensiones y descripción, se muestra a continuación:

Propiedades: sustitución de las variables $x$ $e y$, por números enteros que cumplan las condiciones y hagan verdadera la ecuación.

Dimensión: números enteros positivos o negativos asignados a las variables $x$ e $y$ que cumplan una condición determinada.
Descripción: formas de especificar y relacionar números enteros que sustituyan las variables $x$ e $y$, haciendo verdadera la ecuación.

El segundo código. Ante la cuestión 3, acerca de ¿qué relación o qué condiciones deben cumplirse para la ecuación sea verdadera?, P1, manifestó:

El valor que se encuentre de " $x$ " $y$ " $y$ " debe adicionarle a uno y disminuirle al otro. Pero, no a ambos valores disminuirles $\mathrm{o}$ adicionarles al mismo tiempo.

Interpretación y sentido dado a los datos. $P 1$, establece una relación clara, acerca de las condiciones que deben cumplir la dupla de números enteros que hacen verdadera la ecuación: mientras uno aumenta el otro debe disminuir o viceversa.

Mientras que la participante $\mathrm{P} 2$, ante la misma cuestión, afirma:

Debe existir un Divisor que divida a los valores de $a, b$ y $c$.

Interpretación y sentido dado a los datos. La estudiante $P 2$, establece una condición necesaria, sobre los coeficientes $a, b$ de las variables $x e y$, con el término independiente $c$.

Por su parte el participante, codificado como P5, manifiesta:

Que los valores de $x$ y yaumentan o disminuyen proporcionalmente a un valor que depende de la pendiente.

Interpretación y sentido dado a los datos. La afirmación de P5, se debe a que la cuestión 1 , sugería que se reemplazaran las variables $x$ e $y$ por números que hicieran verdadera la ecuación, P5 le dio un tratamiento algebraico a la ecuación, despejando la variable $y$, además 
de interpretar la ecuación como una función.

El código establecercondiciones. A continuación, se muestran sus propiedades, dimensiones y descripción:

Propiedades: afirmaciones acerca de las relaciones que deben cumplir los números enteros $a, b y c$ o las variables $x$ e $y$ que hagan verdadera la ecuación.

Dimensión: tipos de afirmaciones acerca de los nexos entre las constantes $a, b$ y $c$, las variables $x$ e $y$ que hacen verdadera la ecuación.

Descripción: formas en que cada participante elabora y establece relaciones entre las constantes $a, b$ y $c$, las variables $x$ e $y$, que hacen verdadera la ecuación.

Fase 2. El proceso simultáneo de recolección y análisis de datos continua. Algunos de los códigos iniciales se mantienen en el proceso de codificación y se convierten en categorías, otros son absorbidos por otros conceptos, incluso algunos pueden desaparecer. Esto se debe, a que el investigador se aleja un poco de los datos y empieza el proceso de búsqueda de similitudes y diferencias entre los conceptos iniciales; característica que identifica el proceso de codificación axial.

Ante el problema: Sí en la ecuación lineal diofántica $a x+b y=c$ se exige que las

soluciones sean exclusivamente en los enteros negativos. ¿Qué condiciones deben imponerse sobre las constantes $a, b$ y $c$, para cumplir con esta exigencia?

Las acciones manifestadas por el estudiante codificado como SA P1:

Si decimos que los valores de $(x, y)$ deben ser enteros negativos, las condiciones a plantear con respecto para son las siguientes: $\quad(a, b) \in \mathbb{Z}^{+}$ debe ser cualquier valor arbitrario entero pero positivo $c \in \mathbf{Z}^{-}$ si se plantea que $(x, y) \in \mathbb{Z}^{-}$ y $(a, b) \in \mathbb{Z}^{+}$

el valor de $c$ siempre será negativo.

Las soluciones particulares $(x, y)$ deben ser menor a (0), es decir $x<0$ y $y<0$

El mismo participante, afirma:

Se describen otras condiciones para que los valores de $(x, y) \in \mathbb{Z}^{-}$ $a \in \mathbb{Z}^{-}$debe pertenecer a los enteros negativos $b \in \mathbb{Z}^{+}$debe pertenecer a los enteros positivos

Se establece el valor de $c$ de dos formas $c \in \mathbb{Z}^{-}$si el producto de by $>a x$

$$
c \in \mathbb{Z}^{+} \text {si el producto de } a x>b y
$$

De esta manera $(x, y) \in \mathbb{Z}^{-}$

\section{Interpretación y sentido dado a los datos.} Las acciones manifestadas por P1, evidencia la manera cómo, desde la variación y el cambio, el participante: primero establece una serie de relaciones y condiciones generales, sobre los valores de los números enteros $a, b$ y $c$, para cumplir con las exigencias del problema y la manera, cómo a su manera explica y justifica estas afirmaciones.

Como se puede observar a estas alturas del proceso, el estudiante $\mathrm{P} 1$, muestra un razonamiento mejor elaborado, esto, como producto de su trabajo en las dos secuencias de aprendizaje. Estas acciones y las de los demás participantes, confirmaron los códigos 
combinar y establecer condiciones, además se construyeron nuevos códigos que se denominaron: asignar, formalizar, generalizar y probar.

Ante la cuestión: Plantee (invente) y solucione un problema de la vida real que involucre una ecuación lineal diofántica $a x+b y=c$

donde $x$ e $y$ sean ambos enteros positivos, que tenga infinitas soluciones. El participante P5, plantea el problema:

\section{El séptuple de un número entero menos el triple de otro entero es igual a 22. ¿Cuáles son esos pares de enteros positivos que satisfacen la condición anterior?}

Interpretación y sentido dado a los datos. P5, plantea este problema con seguridad debido a que las cuestiones y sugerencias trabajadas hasta el momento, le habían permitido establecer condiciones necesarias y suficientes, como: el máximo común divisor de , debe dividir a , expresado matemáticamente sería,

$$
\operatorname{mcd}(7,-3)=1 \mid 22
$$

Otra condición que el participante había elaborado es, que mientras el valor de una variable aumenta, el valor de la otra disminuye o viceversa.

El estudiante utiliza el método de fracciones continuas para resolver el problema. Obtuvo las siguientes expresiones algebraicas que le permitieron hallar las infinitas soluciones:

$$
x=22-3 t, \quad y=44-3 t, \quad \text { con } t \in \mathbb{Z}
$$

Como se exigen soluciones en los enteros positivos, P5 plantea:

$$
x=22-3 t>0, \quad y=44-3 t>0
$$

realiza un trabajo de manipulación de expresiones algebraicas entre desigualdades, para concluir que:

$$
t<6,28 \text {, con } t \in \mathbb{Z}
$$

Reconoce que los números enteros dados a $t$,que cumplan esta relación, determinan las infinitas soluciones en los números enteros positivos al problema dado. Es decir, se establecen nuevas condiciones, cuando se exigen algunas restricciones sobre los tipos de soluciones requeridas.

Ante la pregunta, ¿cómo determinar las infinitas soluciones al problema, el participante P5, afirma:
A partir de las condiciones del problema y por los valores de " $t$ " podemos concluir que son infinitas las soluciones de encontrar en enteros positivos tal que el séptuple del primero menos el triple del segundo sea igual a .

\section{Interpretación y sentido dado a los datos.} Luego de resuelto el problema, y su trabajo en las ecuaciones que determinan los valores de $x$ e $y$, el estudiante concluye que los valores que puede tomar $t \in \mathcal{Z}$ determinan el tipo de solución exigida.

\section{El código, $t \in \mathbb{Z}$ determina el tipo de} soluciones. A partir del análisis y sentido a los datos se especifican sus propiedades $y$ dimensiones:

Propiedades: tipos de condiciones en el planteo del problema a las que debe responder $t$

Dimensiones: rango de variabilidad de los números enteros que puede asumir $t$ para cumplir las condiciones impuestas en el planteo del problema. 
Descripción: tipos de condiciones impuestas en el planteo del problema que determinan el rango de variabilidad de los valores de $t$.

Se empieza a vislumbrar aquí una relación en la que las condiciones que se exijan al plantear la ecuación lineal diofántica de la forma

$$
a x+b y=c \text { de alguna manera tienen }
$$

nexos con el problema planteado. En el proceso de análisis y búsqueda de relaciones entre categorías, se siguió a Corbin y Strauss (2017). Estos autores recomiendan y sugieren que al examinar los datos y los primeros códigos en la búsqueda de categorías deben relacionarse las condiciones causales, el contexto, las condiciones de intervención, las estrategias de acción- intervención y las consecuencias.

Fase 3. La secuencia de aprendizaje 3, fue implementada y las entrevistas no estructuradas fueron realizadas a cinco participantes. Los problemas propuestos fueron más retadores para los participantes, retadores en el sentido que requieren intuición, creatividad e ingenio del solucionador. Se pidió también, que plantearan problemas matemáticos con ciertas exigencias en la ecuación lineal diofántica, así como plantear problemas de la vida real que involucren este tipo de ecuación. A continuación, se transcribe literalmente, apartes de la entrevista con el participante P1 y PI que significa profesor investigador:

PI: suponga que usted es profesor y está orientando a los estudiantes en este tema, ¿qué variaciones o haría o qué condiciones impondría a la ecuación $a x+b y=c$

de tal manera que sea un reto, como usted lo ha vivido y lo está trabajando, para que lleve al estudiante a pensar matemáticamente?
P1: En este..., pues la pregunta es sencilla, pero fue un poco complicado inventar esas variaciones, ya que en toda la temática del desarrollo ha sido un verdadero reto encontrar esas respuestas a las actividades que ha planteado, haciendo un esfuerzo cognitivamente de nosotros, interpretando las preguntas y estableciendo relaciones. Para mí lo que ha establecido ya es un reto y son variaciones que debe tener en cuenta por eso se me dificultaría esta pregunta, en la cual yo respondí que para plantear una fórmula donde se relacionen los valares de $a$ y $b$, se aplique el valor de c, es decir decirle al estudiante que me genere esa fórmula establecida al inicio y me diga qué relación, justificando claro esta demostrativamente que relación se establece entre los valores de a y bpara obtener un valor de $c$. Una segunda es establecer un número de soluciones en los enteros positivos que satisfaga para cualquier límite de soluciones, es decir no limitarla a un valor numérico ya sea tres, cuatro, cinco, seis o siete, sino que cumpla para todas, que él me haga un razonamiento crítico donde el me demuestre, compruebe y justifique que esa me puede servir para todas, pues haciendo el método de generalización y comprobando con algunos ejemplos y que me pueda cerciorar de que eso es cierto. Otra sería de qué forma debe estar la ecuación $a x+b y=c$ 
ya que ella puede tomar cuando a es negativo, cuando $b$ es negativo o cuando ambas son negativas para obtener infinitas soluciones en los enteros positivos y negativos $y$ de la misma forma justificar la respuesta $y$, por último, establecer una relación entre los productos de $a x y$ by

para que el valor de torne a positivo o negativo y cuál es la relación entre de esos dos productos, y pues como puede observar en casi todas, siempre va a quedar una parte, dígala o no la mencioné, en las variaciones que es de justificar, ya que justificar es lo que más se nos dificulta, es establecer un razonamiento, estableciéndolo con palabras, puede ser una parte tedioso que se puede complicar a cualquier estudiante, inclusive para mí, pues a mí la justificación se me complica y por eso la establezco como uno de los criterios.

Interpretación y sentido dado a los datos. El participante $\mathrm{P} 1$, propone una serie de variaciones a imponer a la solución de la ecuación lineal diofántica $a x+b y=c \quad$ estas nuevas exigencias desde su forma de pensar son un verdadero reto para el solucionador del problema. De la misma manera, $\mathrm{P} 1$, aunque no lo hace explicito, manifiesta la relación que debe haber entre el razonamiento al solucionar el problema, su justificación y la forma de prueba.

El código, variar condiciones a la solución exigida. Como estrategia para reformular el problema de resolver la ecuación lineal diofántica $a x+b y=c$
Propiedades: variaciones o exigencias en el tipo de solución requerida a la ecuación lineal diofántica $a x+b y=c$

Dimensiones: tipos de condiciones y/o exigencias impuestas las soluciones de la ecuación

$a x+b y=c$

Descripción: variación de condiciones y/o exigencias impuestas a la solución de la ecuación lineal diofántica $a x+b y=c$

Siguiendo con los apartes de la entrevista, ante la pregunta o cuestión siete:

PI: ¿qué recomendaciones?, siguiendo la cuestión 7: enuncie y escriba una serie de pasos - acciones para que alguien los pueda seguir y plantear o inventar problemas de la vida real relacionados con las ecuaciones lineales diofánticas de la forma $a x+b y=c$ ?, puesto que usted planteó o inventó problemas, sus compañeros también lo hicieron, entonces, ¿qué recomendaría usted?:

P1: Primeramente, para establecer una problemática, primero tenemos que encontrar la ecuación, en la que vamos a sumergir esa problemática. Yo, primeramente, coloco establecer la ecuación diofántica que cumpla las condiciones anteriormente explicadas, [el participante hace referencia a la respuesta que aparece en párrafos anteriores] luego de eso, elegir el contexto en el que se desea ejecutar, teniendo en cuenta que $x$ y $y$ sean positivos $o$ negativos o que se intercambien. 
Porqué lo digo de esa forma, porque hay varios contextos donde una persona adquiere productos y al vender adquiere monetización, es decir adquiere dinero. Hay varios, teniendo en cuenta también ejercicios en deportes y demás donde los valores de $x$ y $y$, no van a ser todos positivos, pueden tender a ser negativos, pérdida de un producto, estableciendo con intereses de préstamo, hay varios contextos, entonces eso es lo puntual que tiene que tener en cuenta. Después verificar que los valores de $a, b$ y $c$ sean acordes al contexto, no meter valores este, técnicamente absurdos, donde digamos alguien va a fabricar unos vestidos o un tipo de prenda de vestir y saben que se demoran esos vestidos en crearse, digamos por la semana manejados por una persona, como ejemplo, perdón que si me detengo, que cree diariamente 2 y, eso, a mucho esfuerzo a la semana crearía 14, ósea obtener los valores de $x$ y $y$, donde me vayan a decir que 88,115 o 600 o por allá infinitamente, sería absurdo, es decir que la problemática sea acorde a esos valores y no absurdamente imposibles. Luego, pues creo unos contextos, pues aquí están contaduría, dinero, mercancía de productos, datos según el tiempo, deportes, a tener en cuenta, y ya como parte final, si se desea plantear, pues si lo voy a colocar con una dificultad mayor, pues establezco una dificultad menor, si es plantear un problema sencillo nombrar los datos y respectivamente su criterio, es decir establecerles el signo a primera vista, no generar confusión en los valores de $x$ y $y$, y establecer una pregunta corta y que sea específica. Ahora, si queremos que al estudiante de verdad se le dificulte, los datos de a y bse muestran de manera implícita sin especificar el signo, nada mas decir que a y bpertenecen a tal cosa, pues sumergidos en el problema, pero no podemos decir si ellos son positivos o negativos, nada mas se establecen y con una lectura crítica él tenga que justificar que el signo es acorde. Establecer las incógnitas $x$ y $y$, pero, sin determinar también el signo, es decir podemos tomar palabras exportar, importar, sustraer, retener, devolver, intereses, acreditar, interés de mora o entre otras palabras, que sean difíciles para la comprensión, donde él tenga que leer detenidamente, buscar esos significados, sí él no los conoce para poder establecer esa ecuación adecuadamente, luego establecer una pregunta poco particular donde se tenga en cuenta el lector que los días de trabajo, tiempos establecidos valores creíbles, relación válida entre estos valores, es decir, si el trabajador.

Interpretación y sentido dado a los datos. El participante P1 y sus compañeros coinciden en que lo primero que se debe hacer para plantear un problema de la vida real que involucre ecuaciones lineales diofánticas de la forma $a x+b y=c$ es establecer o imponer 
condiciones sobre la ecuación, como por el ejemplo el tipo de soluciones o condiciones sobre los números $a, b$ y $c$, posteriormente teniendo en cuenta el tipo soluciones exigidas buscar y elegir el contexto o situación del problema.

Propone algunos contextos donde las soluciones sean enteros positivos onegativos, o se intercalen los signos de $x$ e $y$, como por ejemplo compra de productos, pérdida de un producto, intereses, datos según el tiempo y deportes, entre otros. Estas condiciones las establece puesto que, ya había resuelto problemas donde debió buscar relaciones entre los elementos de la ecuación que cumplieran determinadas condiciones para responder a un tipo de solución. Manifiesta que los números $a, b$ y cno pueden ser cualquier número, deben ser acordes al contexto, además de ser números enteros.

El código, establecer condiciones sobre la solución exigida a la ecuación lineal diofántica

contextos de la vida real.

Propiedades: condiciones exigidas al tipo de solución de la ecuación lineal diofántica para determinar los contextos de la vida real.

Dimensiones: tipos de soluciones impuestas a las variables $x$ e $y$ como solución de la ecuación que determinan los contextos del problema a plantear.

Descripción: variedad de condiciones impuestas a la solución a la ecuación lineal diofántica $a x+b y=c \quad$ que determinan los contextos de la vida real para plantear el problema.

\section{RESULTADOS}

Como resultado de los procesos simultáneos de recolección, análisis y codificación, muestreo y saturación teórica surge el núcleo de la teoría, que se denominó: estrategias en el planteo y resolución de problemas que involucran ecuaciones lineales diofánticas de la forma $a x+b y=c$

La Tabla 2, muestra esta categoría, las subcategorías que la integran junto a su descripción.

Tabla 2. Categoría central acerca del planteo y resolución de problemas

\begin{tabular}{|c|c|c|}
\hline Categoria & Subcategoria & Descripción \\
\hline \multirow{2}{*}{ Conjeturar } & Combinar & \multirow{2}{*}{$\begin{array}{l}\text { Formas verbales o escritas de expresar relaciones } \\
\text { y condiciones al combinar números enteros que } \\
\text { hagan verdadera la ecuacion. }\end{array}$} \\
\hline & Establecer condiciones & \\
\hline \multirow{2}{*}{ Generalizar } & Asignar & \multirow{2}{*}{$\begin{array}{l}\text { Formas de utilizar letras, signos y expresiones } \\
\text { matemáticas para formalizar relaciones entre los } \\
\text { diferentes elementos que conforman el problema. }\end{array}$} \\
\hline & Formalizar & \\
\hline \multirow{2}{*}{$\begin{array}{l}\text { Probar } \\
\text { inductivamente }\end{array}$} & Elemplos & \multirow{2}{*}{$\begin{array}{l}\text { Formas de utizar ejemplos y contraejemplos para } \\
\text { convencerse a si mismo y convencer a los demas. }\end{array}$} \\
\hline & Contra ejemplos & \\
\hline \multirow[b]{2}{*}{$\begin{array}{l}\text { Plantear } \\
\text { problemas }\end{array}$} & $\begin{array}{l}\text { Reformular condiciones } \\
\text { a las infnitas soluciones } \\
\text { de ELD } a x+b y=c\end{array}$ & $\begin{array}{l}\text { Formas de imponer nuevas condiciones sobre las } \\
\text { soluciones a la ecuación lineal diofantica de la } \\
\text { forma } a x+b y=c \text {. }\end{array}$ \\
\hline & $\begin{array}{l}\text { Seleccionar situaciones } \\
\text { de la vida real que } \\
\text { comespondan al tipo de } \\
\text { solucion exigida a la } \\
\text { ELD } a x+b y=c\end{array}$ & $\begin{array}{l}\text { Formas de elegir stuaciones de la vida real en } \\
\text { dominios enteros acorde con las condiciones } \\
\text { exigidas a la ELD } a x+b y=c \text { y las relaciones de } \\
\text { los números enteros } a, b \text { y } c \text {. }\end{array}$ \\
\hline
\end{tabular}




\section{CONCLUSIONES Y DISCUSIÓN}

El haber elegido un enfoque cualitativo con un diseño desde la teoría fundamentada, para responder la pregunta: ¿Cómo son las estrategias?, manifestadas por el grupo de participantes en el contexto propuesto, posibilitó la elaboración inductiva de la categoría central o núcleo de la teoría que se denominó: estrategias en el planteo y resolución de problemas que involucran ecuaciones lineales diofánticas de la forma $a x+b y=c \quad$ y sus respectivas

subcategorías.

Desde la teoría fundamentada, los resultados se consideran como un proceso, compuesto por un flujo de acciones e interacciones entre procesos y subprocesos. Es variable por naturaleza, puesto que cada participante lo elaboró y construyó a su manera. Cada proceso está constituido por subprocesos, como se observa en la Tabla 2. Las categorías se construyeron desde los datos (manifestaciones verbales y escritas) siguiendo la evolución en las tareas de los participantes. Aunque las estrategias fueron caracterizadas de esta manera, otros investigadores, desde otra mirada y con otras perspectivas pueden obtener y presentar resultados diferentes.

Los resultados muestran tres elementos fundamentales interrelacionados entre sí. En la resolución de problemas las subcategorías, conjeturar, generalizar y probar son consecuentes y están inmersas en las fases de entrada, ataque y revisión propuestas por Mason, Burton y Stacey (2010), las etapas entender el problema, diseñar un plan y ejecurtarlo, mirando hacia atrás en el trabajo propuesto por Polya (1945) y la dimensión que Schoenlfed (2016) denomina estrategias de resolución. Lo anterior radica, en que las propuestas de estos autores son muy generales, esta investigación se hizo desde un contexto específico: el dominio de los numeros enteros.
En lo que respecta al planteo de problemas, los resultados muestran que la estrategia de imponer nuevas condiciones a las infinitas soluciones de la ecuación lineal diofántica de la forma $a x+b y=c$ origina un nuevo problema para el solucionador; esto coincide con la estrategia de reformular el problema, como estrategia para plantear nuevos problemas como sugieren muchos investigadores, como Brown y Walter (1983), Silver (2013) entre otros. Estas nuevas exigencias, junto a las estrategias de conjeturar, generalizar y probar posibilitaron a los participantes buscar contextos de la vida real como situaciones para plantear problemas, que se ajusten a estos nuevos requerimientos.

Los resultados muestran y coinciden también en otro aspecto fundamental: desde el contexto de resolver problemas que involucran este tipo de ecuaciones en dominios discretos, el planteo de problemas está inmerso en los procesos de resolución, coincidiendo con autores como Polya (1945), Leung (2016), Felmer, Pehkonen y Kilpatrick (2016). Este aspecto reafirma lo propuesto por Cai y Hwang (2002), Cai y Hwang (2020), Cai, et al. (2015) quienes en sus trabajos encontraron que hay una fuerte relación entre la resolución y el planteo de problemas y que los solucionadores más exitosos fueron quienes plantearon problemas más complejos.

Finalmente los autores consideran que el planteo y resolución de problemas desde dominios discretos es consecuente con lo que afirman Felmer, Pehkonen y Kilpatrick (2016), en el sentido de que enriquecen el trabajo de los estudiantes puesto que las altas exigencias cognitivas, requieren de ingenio y creatividad a quien resuelve y plantea problemas. Queda abierta la posibilidad hacer estos trabajos desde otros escenarios, por ejemplo desde dominios discretos en situaciones geométricas. 


\section{REFERENCIAS BIBLIOGRÁFICAS}

Brown, S., \& Walter, M. (1983). The art of problem posing. Hillsdale, $\mathrm{NJ}$ : Lawrence Erlbaum Associates.

Cai , j., \& Hwang, S. (2020). Learning to teach through mathematical problem posing: Theoretical considerations, methodology, and directions for future research. International Journal of Educational Research, 102, 10391.

Cai, J., \& Hwang, S. (2002). Generalized and generative thinking in US and Chinese students' mathematical problem solving and problem posing. The Journal of mathematical behavior, 21(4), 401-421.

Cai, J., Chen , T., Li, X., Xu, R., Zhang, S., Hu, Y., . . . Song , N. (2020). Exploring the impact of a problem-posing workshop on elementary school mathematics teachers' conceptions on problem posing and lesson design. International Journal of Educational Research, 102, 101404.

Cai, J., Hwang, S., Jiang, C., \& Silber, S. (2015). Problem-posing research in mathematics education: Some answered and unanswered questions. En Mathematical problem posing (págs. 3-34). New York, NY: Springer.

Cai, J., Jiang, C., Hwang, S., Nie, B., \& Hu, D. (2016). How do textbooks incorporate mathematical problem posing? An international comparative study. En Posing and solving mathematical problems (págs. 3-22). Springer.

Charmaz, K. (2006). Constructing grounded theory: A practical guide through qualitative analysis. London: Sage Publications.
Charmaz, K. (2014). Constructing grounded theory (2 ed.). Thousand Oaks, CA: Sage.

Corbin, J., \& Strauss, A. (2008). Basics of qualitative research:Techniques and procedures for developing grounded theory (3 ed.). Thousand Oaks, CA, USA: SAGE Publications.

Corbin, J., \& Strauss, A. (2017). Conceptos básicos de la investigación cualitativa: técnicas y procedimientos para desarrollar la teoría fundamentada (4 ed.). Thousand Oaks, California, United States of America: SAGE Publications.

Ellerton, N., Singer, N., \& Cai, J. (2015). Problem Posing in Mathematics: Reflecting on the Past, Energizing the Present, and Foreshadowing the Future. En F. Singer, N. F. Ellerton, \& J. Cai (Edits.), Mathematical Problem Posing. Research in Mathematics Education. New York, NY: Springer. doi:https://doi. org/10.1007/978-1-4614-6258-3_26

Ernest, P. (1997). Chapter 3: The epistemological basis of qualitative research in mathematics education: A postmodern perspective. Journal for Research in Mathematics Education. Monograph, 9, 22-177. doi:10.2307/749945

Felmer, P., Pehkonen, E., \& Kilpatrick, J. (2016). Posing and Solving Mathematical Problems. Springer International Publishing. doi:https://doi. org/10.1007/978-3-319-28023-3

Ginsburg, H. (1981). The clinical interview in psychological research on mathematical thinking: Aims, rationales, techniques. For the learning of mathematics, 1(3), 4-11. 
Kilpatrick, J. (1987). Problem formulating: Where do good problems come from? En A. Schoenfeld, \& A. Schoenfeld (Ed.), Cognitive science and mathematics education (págs. 123-147). Hillsdale, $\mathrm{NJ}$ : Lawrence Erlbaum Associates.

Kupisiewicz, C. (1964). O efektívnosti problémového vyučovania: výskum vyučovacích metód matematickoprírodovedných predmetov.

Leung, S. (2016). Mathematical problem posing: A case of elementary school teachers developing tasks and designing instructions in Taiwan. En Posing and solving mathematical problems (págs. 327-344). Cham: Springer.

Mason, J., Burton, L., \& Stacey, K. (2010). Thinking Mathematically (2 ed.). Harlow, UK: Pearson Education Limited.

Mayer, R. (2010). Problem Solving and Reasoning. International Encyclopedia of Education, 273-278. doi:10.1016/ B978-0-08-044894-7.00487-5

Polya, G. (1945). How To Solve It. Princeton: Princeton University Press.

Polya, G. (1981). Mathematical Discovery. New York: Wiley.

Schoenfeld, A. H. (2016). Learning to think mathematically: Problem solving, metacognition, and sense making in mathematics (Reprint). Journal of Education, 196(2), 1- 38. doi:10.1177/002205741619600202

Silver, E. (2013). Problem-posing research in mathematics education: Looking back, looking around, and looking ahead. Educational Studies in Mathematics, 83(1), 157-162.

Von Glasersfeld, E. (2013). Radical constructivism (Vol. 6). Routledge.

Xu, B., Cai, J., Liu, Q., \& Hwang, S. (2019). Teachers' predictions of students' mathematical thinking related to. International Journal of Educational Research. doi:doi:10.1016/j. ijer.2019.04.005 